\title{
Rarely Described Renal Malignancies Associated With Venous Tumor Thrombus
}

\author{
MARIA C. VELASQUEZ ${ }^{1,2}$, ALI MOUZANNAR ${ }^{1,2}$, SANJAYA SWAIN ${ }^{1,2}$ and GAETANO CIANCIO ${ }^{1,2,3}$ \\ ${ }^{1}$ Department of Urology and Surgery, Jackson Memorial Hospital, Miami, FL, U.S.A.; \\ ${ }^{2}$ University of Miami Miller School of Medicine, Jackson Memorial Hospital, Miami, FL, U.S.A.; \\ ${ }^{3}$ Miami Transplant Institute, Jackson Memorial Hospital, Miami, FL, U.S.A.
}

\begin{abstract}
Background/Aim: Collecting duct carcinoma, epithelioid angiosarcoma and neuroendocrine/carcinoid tumor are uncommon renal malignancies, and their association with tumor thrombus extending into the inferior vena cava is extremely rare. Owing to the rarity of the above-mentioned malignancies and short follow-up of the cases published in the literature, the prognosis and clinical behavior of these tumors remains unclear. Up to date, the culprit of treatment is surgical management with radical nephrectomy, lymph node dissection, thrombectomy and vascular reconstruction if necessary. Patients and Methods: We herein describe in detail the first cases published of the above-mentioned renal malignancies associated with extensive inferior vena cava (IVC) thrombus, in which complex vascular reconstruction was performed Results: Three male patients were identified as having collecting duct carcinoma, epithelioid angiosarcoma and neuroendocrine/ carcinoid tumor with IVC involvement. Tumor thrombus levels were II, I and IIIc respectively. Patient ages were 42, 60 and 47 years and tumor sizes were 9.2, 10.9 and $3.7 \mathrm{~cm}$ correspondingly. Patient 2 underwent cavectomy, IVC replacement using polytetrafluoroethylene (Gore-Tex ${ }^{(B)}$ vascular graft and IVC filter deployment inside the graft. None of the patients developed any pulmonary emboli postoperatively. At the last follow-up, IVC graft for patient 2 remained patent. Conclusion: Owing to the rarity of the aforementioned malignancies and short follow-up of cases
\end{abstract}

Correspondence to: Gaetano Ciancio, MD, Department of Urology, Jackson Memorial Hospital, 1801 NW $9^{\text {th }}$ Ave, $7^{\text {th }}$ Floor, Miami, FL 33136, USA. Tel: +1 3053555803, Fax: +1 3053555797, e-mail: gciancio@med.miami.edu

Key Words: Renal carcinoma, tumor thrombus, surgical reconstruction, inferior vena cava.

This article is an open access article distributed under the terms and conditions of the Creative Commons Attribution (CC BY-NC-ND) 4.0 international license (https://creativecommons.org/licenses/by-nc-nd/4.0). published in the literature, the prognosis and clinical behavior of these tumors remains unclear. Up to date, the culprit of treatment is surgical management with radical nephrectomy, lymph node dissection, thrombectomy and vascular reconstruction if necessary. Polytetrafluoroethylene (Gore-Tex) vascular grafts are an excellent and safe option for complex vascular reconstructions in patients with evidence of IVC invasion.

Renal cell carcinoma (RCC) comprises a heterogenous group of malignant neoplasms, with more than a dozen recognized histological variants, accounting for nearly $90 \%$ of all renal malignancies. The most common type of RCC is clear cell, followed by papillary and chromophobe; which represent $85 \%$ of all RCC cases (1). The remaining $15 \%$ comprises rarer renal tumors such as collecting duct carcinoma (CDC); which accounts for only $<1 \%$ of all renal cell carcinomas and is characterized by its aggressiveness, poor prognosis and lack of response to conventional therapies (2). CDC arises from the Bellini ducts epithelium, located in the distal collecting duct of the renal medulla, and was first reported by Foot and Papanicolaou in 1949. On the other hand, primary renal epithelioid angiosarcoma; which mimics epithelial tumors, both morphologically and immunohistochemically, is an extremely rare and aggressive malignancy with a rapidly metastatic and fatal course, which presents a great diagnostic challenge as it mimics RCC, and it is associated with rapid local and bloodstream dissemination. Consequently, patients usually have advanced disease when diagnosed and a high risk for recurrence (3). Lastly, primary well-differentiated renal neuroendocrine tumors (carcinoid) area also exceedingly rare neoplasms, considered a distinct entity within the latest World Health Organization Classification scheme (4), with histological features identical to carcinoid tumors found at other anatomical locations (5). Within the kidney, carcinoid tumors have been reported to arise most commonly from horseshoe kidneys $(6,7)$, with previous reports documenting early developing of metastatic disease and poor patient outcomes with increased mitotic 
activity and cytologic atypia within the renal carcinoid tumor (5). In this article, we present three patients with rare renal malignancies associated with tumor thrombus extending into the inferior vena cava (IVC). To our knowledge, these are the only cases published in the literature.

\section{Patients and Methods}

This study was performed according to the ethical standards of the University of Miami Institutional Review Board and Declaration of Helsinki (as revised in 2013). Three patients were referred to Jackson Memorial Hospital for treatment of suspected RCC with TT extending into the IVC. The cranial extent of the tumor was defined per our own classification (8), as well as per Neves and Zincke classification (9). Transesophageal echocardiography (TEE) was used to detect any pulmonary emboli before, during and at the end of the three surgical interventions, in order to carefully manage pulmonary emboli. Complex vascular reconstruction was performed as needed.

\section{Case Presentation}

Patient 1. A 42-year-old Hispanic male with past medical history of recurrent nephrolithiasis, was transferred from an outside hospital with a diagnosis of a large left renal mass and bilateral pulmonary emboli. Patient reported unintentional weight loss, anorexia, and intermittent gross hematuria over the last 2 months. Upon arrival to the Emergency Department, computed tomography revealed a $9.2 \times 5.3 \mathrm{~cm}$ left upper pole mass extending to the posterior abdominal wall, multiple left renal hilar and peri-aortic lymph nodes were seen. No venous thrombosis was appreciated, renal vein was patent, and no distant metastasis were identified (Figure 1). Laboratory investigation revealed microscopic hematuria ( $>25 \mathrm{RBC} / \mathrm{HPF}$ ), however complete blood count (CBC) and complete metabolic panel (CMP) were within normal limits. Patient underwent open left radical nephrectomy with lymphadenectomy, IVC mobilization, IVC thrombectomy and IVC reconstruction, given that a level II IVC tumor thrombus was identified intra-operatively.

Patient 2. A 60-year-old Caucasian male with past medical history significant for well controlled essential hypertension and psoriasis, presented to the Emergency Department with a 4-month history of low back pain and unintentional weight loss of 30 pounds. Patient denied gross hematuria, urinary symptoms, smoking history or radiation exposure. At the time of admission, a lumbar magnetic resonance imaging (MRI) was performed, which revealed a large right-sided renal mass distorting the normal renal architecture, with dilation of the renal pelvis and calyceal system, measuring $10.9 \times 9.7 \mathrm{~cm}$. Additionally, abnormal lesions were seen in three vertebral bodies, concerning for metastatic disease. Given the above-mentioned findings, a contrast enhanced computed tomography (CT) scan was performed to better characterize the renal lesion, which revealed a heterogenous right renal mass with a large lymph node located on top of the vena cava, with no identifiable planes between the vena cava and the renal mass. Of note, at the time of admission, patient's creatinine was slightly elevated $(1.6 \mathrm{mg} / \mathrm{dl})$, however hemoglobin and urinalysis were with-in normal limits. Subsequently, patient underwent open right radical nephrectomy with extensive lymphadenectomy, cavectomy, IVC replacement using polytetrafluoroethylene (Gore-Tex ${ }^{\circledR}$ ) vascular graft (W.L. Gore \& Associates, Flagstaff, AZ, USA) and IVC filter deployment inside the graft.

Patient 3. A 47year-old Haitian male with resolved COVID19 infection, was admitted to the Emergency Department with right-sided chest pain, shortness of breath and chills. Patient denied hematuria or abdominal pain, however reported unintentional weight loss and anorexia. At the time of admission, patient was found to be hemodynamically stable, afebrile and with adequate oxygen saturation levels on room air. A contrast enhanced computed tomography (CT) of the chest was performed, which revealed a right hilar mass-like soft tissue density measuring $3.7 \times 2.4 \mathrm{~cm}$ and an incidental large left renal mass. Given the above-mentioned results, an abdominal magnetic resonance imaging (MRI) was ordered, which showed an heterogenous enhancing lesion in the left kidney, extending into the renal vein and inferior vena cava, measuring approximately $12 \times 12.7 \times 9 \mathrm{~cm}$. Tumor thrombus was appreciated throughout the IVC from the level of the renal vein, extending superiorly up to the level of the hepatic vein. No abdominal lymphadenopathy was seen. Laboratory data revealed leukocytosis $\left(18.4 \times 10^{9} / \mathrm{l}\right)$ with normal creatinine and hemoglobin. Urinalysis was negative for hematuria. Pulmonology conducted a bronchoscope exam and found no endobronchial component of the hilar mass and bronchial wash was sent for analysis, which only showed few abnormal cells of epithelial origin. Patient therefore underwent bronchoscopy with endobronchial ultrasound and hilar mass biopsy, which was negative for malignancy, findings were consistent with lymph node tissue. After appropriate surgical clearance was obtained from Pulmonology and Thoracic surgery, patient underwent open left radical nephrectomy, lymph node dissection, en bloc mobilization of liver, pancreas, and spleen, IVC thrombectomy, IVC reconstruction and placement of IVC filter.

Operative technique. A sub-costal incision is performed commencing approximately 2 fingerbreadths below the right or left (according to the tumor location) costal margin, extending out laterally to the mid-axillary line. A Rochard or Thompson self-retaining retractor is placed elevating the costal margins and splaying them laterally towards the axillae. This exposure enables easier liver mobilization, or 


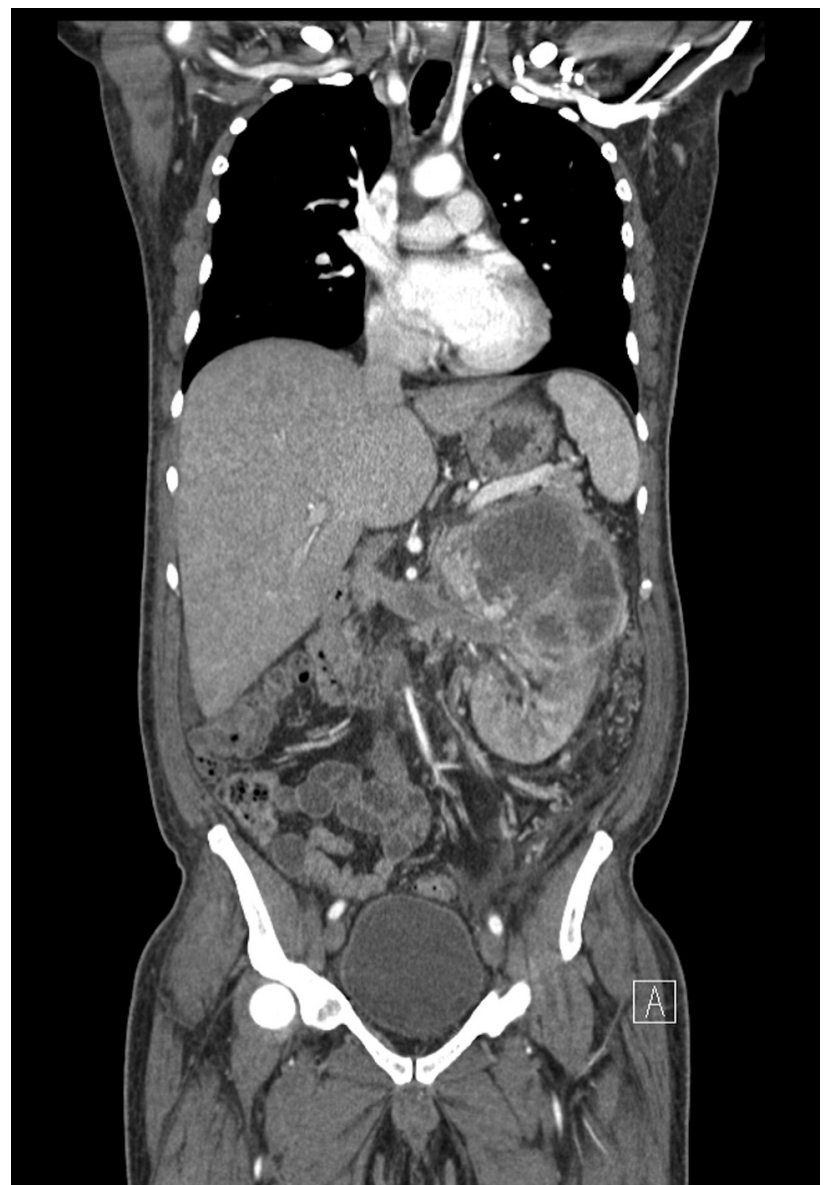

Figure 1. Coronal computed tomography demonstrating a left renal collecting duct carcinoma with level II tumor thrombus (yellow arrow).

en bloc mobilization of pancreas and spleen, as well as access to the retro-hepatic space. Early intraoperative ligation of the involved renal artery is always the main principle of surgical treatment; therefore, the kidney is then mobilized medially, and the renal artery is identified, ligated and divided. Once the renal artery has been ligated, the collateral circulation collapses, facilitating the subsequent resection and decreasing blood loss and need for blood transfusion (10).

For patient 1 , upon mobilizing the left renal vein, it was noted that there was a level II IVC tumor thrombus. The IVC was then mobilized and clamped proximally and distally with vascular clamps. Subsequently, the left renal vein was incised, and the tumor thrombus was carefully removed. The IVC was then over-sewed using 4-0 Prolene in a running fashion.

For patient 2, a more complex IVC reconstruction was performed, as the large right renal mass was encasing almost $70 \%$ of the IVC, associated with a level I tumor thrombus (Figure 2). For this surgical intervention, the liver was completely mobilized off the IVC using an organ transplantbased approach, with the only remaining structural attachments being the hepatic veins and the porta hepatis; a maneuver known as piggyback liver mobilization $(11,12)$. Furthermore, a plane was created between the IVC and posterior abdominal wall, facilitating circumferential control of the IVC. Once the IVC was dissected enough, vascular clamps were placed proximally and distally, including normal IVC for the vascular reconstruction. A vascular clamp was also placed at the level of the left renal vein. Subsequently, the IVC was transected $1 \mathrm{~cm}$ below the renal vein and a $3 \mathrm{~cm}$ above the bifurcation, as there was significantly tumor infiltration. A $20 \mathrm{~mm}$ Gore-Tex vascular graft was used for the vascular reconstruction of the IVC. The graft was first approximated with 4-0 Prolene suture in a running fashion to the proximal end of the cavectomy and flushed with heparin. Then, a vascular clamp was placed below the left renal vein to allow drainage into the IVC. Finally, the anastomosis between the vascular graft and the distal IVC was performed, with 4-0 Prolene suture in a running fashion manner. Before the anterior portion of the vascular anastomosis was performed, a TrapEase IVC filter was carefully placed under direct visualization. Subsequently, the distal anastomosis was completed, the distal IVC vascular clamp was released to allow bleeding and carefully evaluate if clots were present. The proximal vascular clamp was then removed (Figure 3).

For patient 3 , in which a level IIIc IVC tumor thrombus was identified (8) (Figure 4), a vascular clamp was placed distal to the thrombus. The left gonadal vein was significantly enlarged; therefore, it was also clamped. Before performing the pringle maneuver (previously described), a vascular clamp was placed on the right renal and adrenal veins. Subsequently, the Pringle maneuver was performed to temporarily occlude blood inflow to the liver, before clamping the supra-hepatic IVC at the level of the diaphragm. The IVC was then incised, and the thrombus was removed, however a small portion of the thrombus was invading the IVC wall; therefore, this vascular segment was excised. The vascular clamp was then repositioned closer to the hepatic veins. An IVC filter was then placed under direct visualization above the IVC bifurcation and the IVC was closed with 4-0 Prolene and the distal vascular clamp was removed. Before finishing the case, we carefully palpated the IVC once again, to make sure no tumor thrombus was left behind after releasing the distal clamp. At this point a masslike structure was felt at the level of the filter, therefore the IVC was re-clamped distally and incised. A large clot was seen just distal to the IVC filter, which most likely had dislodged from the internal or external iliac vein. The clot was removed without issues and the IVC was closed using 40 Prolene. The clamp was then released. Lastly, a final IVC examination was performed before finishing the procedure. 


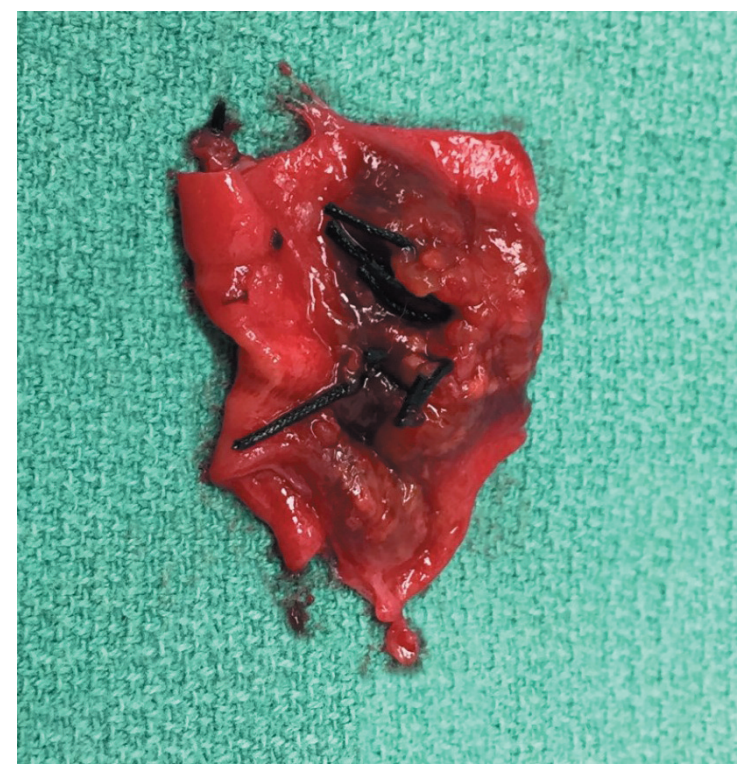

Figure 2. Segment of the inferior vena cava (IVC) infiltrated by epithelioid angiosarcoma (yellow arrow).

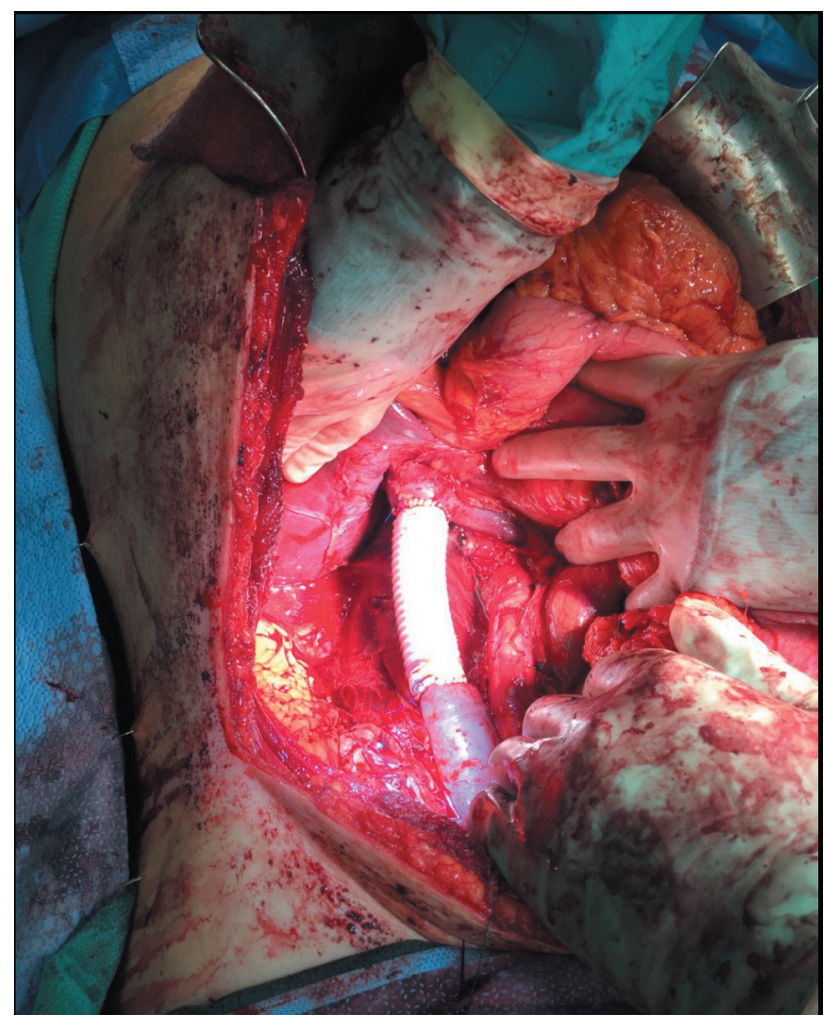

Figure 3. Inferior vena cava (IVC) reconstruction in patient with EAS, using Gore-Tex vascular graft from the left renal vein down to $3 \mathrm{~cm}$ above the IVC bifurcation.

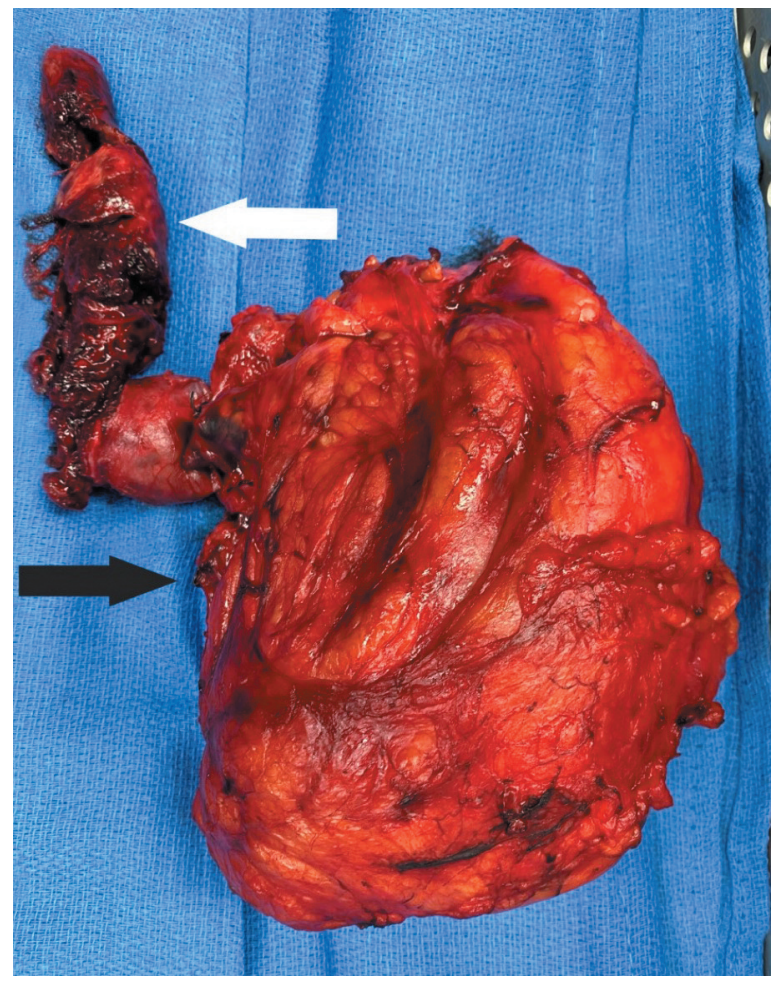

Figure 4. Surgical specimen of large left neuroendocrine renal carcinoma (black arrow) with TT level IIIC extending to the diaphragm (white arrow).

Of note, use of intra-operative TEE was critical to delineate the cranial extent and mobility of the tumor thrombus, during the retro-hepatic or supra-diaphragmatic IVC. Additionally, it provided critical information about the presence of pulmonary embolus or tumor thrombus extending into the right atrium.

All patients were transferred to the Surgical Intensive Care Unit after the surgical intervention for close up monitoring. Patient \#1 and \#3 were extubated on post- operative day (POD) 2, while patient \#2 was extubated immediately after surgery. All three patients had no immediate post- operative complications.

\section{Results}

Operative times were 6,8 and $5 \mathrm{~h}$ for patients 1,2 and 3 respectively. Estimated blood loss was 1,000, 1,000 and 800 $\mathrm{ml}$ for patients 1, 2 and 3 correspondingly. Only patient 1 and 3 required blood transfusion, with 6 and 3 units of packed red blood cells (PRBCs) given intra-operatively to patient 1 and 3 respectively. Patient 2 received $450 \mathrm{cc}$ of Cell-Saver. Final pathology for patient 1 revealed moderately differentiated and extremely necrotic collecting duct carcinoma, for patient 2 a 
Table I. Main patient characteristics

\begin{tabular}{|c|c|c|c|}
\hline & Patient 1 & Patient 2 & Patient 3 \\
\hline \multicolumn{4}{|l|}{ Demographics } \\
\hline Age (years) & 42 & 60 & 47 \\
\hline Gender & Male & Male & Male \\
\hline \multicolumn{4}{|c|}{ Pre-operative diagnostic variables } \\
\hline Laterality & Left & Right & Left \\
\hline Tumor size $(\mathrm{cm})$ & $9.2 \mathrm{~cm}$ & 10.9 & 12.7 \\
\hline Tumor thrombus level & II & I & IIIC \\
\hline \multicolumn{4}{|l|}{ Intraoperative variables } \\
\hline EBL & 11 & 11 & $800 \mathrm{ml}$ \\
\hline Blood transfusion & $6 \mathrm{U}$ & $450 \mathrm{ml}$ of cellsaver & $4 \mathrm{U}$ \\
\hline PTFE graft & No & Yes & No \\
\hline \multicolumn{4}{|l|}{ Outcomes } \\
\hline 30-days complications & None & None & Wound hematoma \\
\hline $\mathrm{CPB}$ & No & No & No \\
\hline OR time (min) & 360 & 480 & 300 \\
\hline LOS (days) & NA & 12 & Inpatient \\
\hline pTNM & pT3aN2M1 & pT2bN0M1 & pT3cNxMx \\
\hline Pathology-variant & Collecting duct carcinoma & Epithelioid angiosarcoma & Neuroendocrine/Carcinoid tumor \\
\hline Histologic grade & $\mathrm{G} 2$ & $\mathrm{G} 3$ & $\mathrm{G} 2$ \\
\hline Lymph nodes (+/total) & $3 / 3$ & $0 / 14$ & NA \\
\hline Pre-operative $\mathrm{Cr}(\mathrm{md} / \mathrm{dl})$ & 0.8 & 1.6 & 1 \\
\hline Post-operative $\mathrm{Cr}$ (md/dl) & 0.7 & 1.2 & 1.5 \\
\hline Follow-up (months) & 24 & 12 & 3 \\
\hline Status at last follow-up & Alive & Deceased from metastatic disease & Alive \\
\hline Anticoagulation & None & NA & Heparin drip \\
\hline
\end{tabular}

EBL: Estimated blood loss; PTFE: polytetrafluroethylene; CPB: cardiopulmonary bypass; OR: operative time; LOS: length of stay; NA: not applicable; Cr: creatinine.

diagnosis was made of renal epithelioid angiosarcoma and for patient 3 final pathology revealed well differentiated neuroendocrine tumor/carcinoid tumor. None of our patients had tumor thrombus above the diaphragm. Serum creatinine after surgical intervention was $0.7,1.25$ and $1.5 \mathrm{mg} / \mathrm{dl}$ for patients 1,2 and 3 respectively. Tumor size was 6,12 and 12.8 $\mathrm{cm}$ for patients 1,2 and 3 correspondingly. The TNM classification was pT3aN2M1 for patient 1 , pT2bN1Mx for patient 2 and pT3cNxMx for patient 3. Patient 1 underwent outpatient chemotherapy and is currently cancer-free. Patient 2 had evidence of metastatic disease upon diagnosis, therefore he underwent chemotherapy treatment at an outside institution, however passed away from the disease one year after the initial diagnosis. Lastly, patient 3 is being followed by the Oncology medical team (Table I).

\section{Discussion}

Clear cell, papillary and chromophobe carcinomas account for $85-90 \%$ of all renal malignancies seen in routine practice. The remaining $10-15 \%$ of renal carcinomas consist of rare sporadic and hereditary tumors, some of which have been rarely described, such as collecting duct carcinoma and specially epithelioid renal angiosarcoma and neuroendocrine tumor/carcinoid tumors $(13,14)$. Collecting duct carcinoma is a rare and aggressive type of RCC, which compromise approximately $1 \%$ of renal epithelial malignancies and usually presents with metastatic disease (13). CDC generally affects patients in the $4^{\text {th }}$ to $7^{\text {th }}$ decade of live, with a male predominance of 2:1. Clinical symptoms ae often nonspecific, and include hematuria, flank pain, palpable abdominal mass and weight loss (2). CDC diagnosis is often based on histopathological findings as tumors may extend into the renal pelvis, mimicking pelvic urothelial carcinoma on imaging studies (15). Typical CT findings include inner medullary location, renal sinus involvement and infiltrative growth that can reach the cortex (2). Microscopic features may be variable, however, the morphologic criteria for diagnosis include: presence of an infiltrative tubular or tubule-papillary pattern associated with a desmoplastic stromal reaction. Additionally, CDC may present with a variety of growth patterns, including tubular, papillary, cribriform and pseudopapillary. Tumor cells typically exhibit high grade nuclear pleomorphism, prominent nuclear atypia and atypical mitotic features $(2,16)$. ACD Immunohistochemical expression typically reveals both high and low 
molecular weight cytokeratins, e-cadherin, peanut lectin, vimentin, c-KIT and Ulex europeaus agglutinin-1, reflecting the origins of the tumor from the collecting duct of the distal nephron. Therefore, markers of proximal renal tubules, such as CD10, RCC antigen and AMACR are always negative $(15,17,18)$. Up to date, different survival studies have shown CDC has a poor prognosis. Pepek et al. (19) published the largest survival analysis up to date, using the Surveillance, Epidemiology, and End Results (SEER) database, and including 98 patients diagnosed with CDC from $1973 v$ to 2004 . Results showed that the Three-year relative survival rates for localized, regional, and distant disease were $93 \%, 45 \%$, and $6 \%$, respectively. Additionally, no significant difference was seen in disease presentation and relative survival based on race and sex. Furthermore, Tokuda et al. (20) published an 81 patient series in which the 1-, 3and 10-year disease-specific survival was 69, 45 and 14\%, respectively. Regional lymph node metastases were present in $44 \%$ of the patients in this series at diagnosis, whereas $32 \%$ had distant metastases. Overall, surgery is the primary treatment for patients with CDC, however in more advanced cases, chemotherapy agents such as gemcitabine and cisplatin are used (21). Targeted therapy, such as sunitinib, sorafenib and everolimus, has also been used with moderate success (22), however due to the aggressiveness of the disease, surgical treatment and chemotherapy are usually not effective in patients with advanced disease, as $2 / 3$ of patients with CDC die within two years after diagnosis (17).

Primary renal epithelioid angiosarcoma (EAS) is an extremely rare and aggressive entity described in the literature by a few case reports. Overall, the etiology is poorly understood, however is well known that it affects primarily white males in the sixth and seventh decades of live, with a male to female ratio of $7: 1$ (23). EAS is an extremely aggressive disease with highly metastatic potential, most commonly to the liver, lungs and bones as the tumor originates from the endothelium of blood and lymphatic vessels. In general the prognosis is grim, with an estimated average life expectancy of 6 months regardless of the presence of metastasis at diagnosis (24). Presenting symptoms are similar to other renal tumors, such as renal cell carcinoma and include flank pain, hematuria, anemia and palpable mass. Other common clinical features include weight loss, anorexia, fever, and general malaise. In general, EAS diagnosis primarily relies on pathological examination, as imaging techniques, such as CT scan and MRI cannot differentiate between EAS and other renal malignancies. Histological examination shows pleomorphic epithelioid cells with vesicular nuclei, prominent nucleoli, and eosinophilic cytoplasm. Given that EAS is often poorly differentiated, the use of immunohistochemical staining is usually required for diagnosis, characteristically staining positive for AE1/AE3, cytokeratin (CK) 7, vimentin, CD31 and Ecadherin, but negative for CD10, CD34, factor VIII, CK20, carcinoembryonic antigen or desmin (25). Given the rarity of this malignancy, up to date there is no standard therapy or treatment guidelines. However, radical nephrectomy is broadly accepted as the mainstay of treatment. Up to date, there are no studies evaluating treatment response to surgery, chemotherapy or radiation exclusively in patients with EAS, though a recent retrospective study of renal angiosarcoma case reports by Iacovelli et al. (26), found that the vast majority of patients (93\%) had a radical nephrectomy as first line treatment, of those, one-third of patients had evidence of metastatic disease. Disease free survival was 6 months (95\% CI=4.3-7.7), compared to 2 months (95\% CI=1.0-3.0) in those patients who underwent chemotherapy as first line treatment.

Lastly, neuroendocrine renal carcinoma (commonly referred to as carcinoid tumors), represents less than $1 \%$ of all primary renal malignancies, occurring both in the renal parenchyma, as well as in the renal pelvis $(27,28)$. Patients with horseshoe kidneys have been reported to have up to 62to 82-fold higher risk of developing neuroendocrine tumors due to abnormal migration of posterior nephrogenic cells (6, 7). Rare cases have also been described in patients with renal teratomas and polycystic kidney disease $(29,30)$. Carcinoid tumors of the kidney are low-grade tumors that tend to grow slowly and can remain silent for a long period of time before causing symptoms. Overall, the prognosis and clinical behavior of renal neuroendocrine tumors remains unclear due to the rarity of this condition, however it seems to be a more indolent malignancy than RCC with better survival rates, even with metastatic disease. Adverse prognostic factors include age greater than 40 years, tumor size greater than 4 $\mathrm{cm}$, mitotic rates higher than $1 / 10$ high power fields, purely solid tumors and metastasis at initial diagnosis (28). Renal carcinoid tumors should be managed by radical nephrectomy, and good outcomes have been obtained for organ-confined disease after radical excision $(27,31)$. Up to date, the biggest case series was published by Jain et al. (32), in which 90 cases reports were reviewed, results showed that there is no gender predilection and age at diagnosis ranged between 23 to 78 years, however the mean age of diagnosis is lower than for RCC. Most of the patients were completely asymptomatic and were incidentally diagnosed. Additionally, Romero et al. (28) reviewed 56 case reports, and found that median patient age was 49 years, incidental diagnosis was made in $28.6 \%$ of patients and metastatic disease was present in $45.6 \%$ of patients at initial diagnosis.

Overall, there is insufficient data regarding surgical techniques for tumor resection in patients with uncommon renal malignancies. However, based on prior experience with most common renal malignancies such as RCC, proper surgical treatment should involve radical nephrectomy, lymph node dissection, tumor thrombectomy and vascular reconstruction if there is evidence of tumoral invasion of vascular structures. Previous studies have shown that polytetrafluoroethylene (Gore-Tex) vascular grafts are an 
excellent and safe option for complex vascular reconstructions in patients with evidence of IVC compromise $(33,34)$. Moreover, IVC vascular reconstruction, regardless of the extent of IVC resection is associated with very low morbidity and low mortality (35).

\section{Conclusion}

Owing to the rarity of the above-mentioned malignancies and short follow-up of the cases published in the literature, the prognosis and clinical behavior of these tumors remains unclear. Up to date, the culprit of treatment is surgical management with radical nephrectomy, lymph node dissection, thrombectomy and vascular reconstruction, if necessary. Polytetrafluoroethylene (Gore-Tex) vascular grafts are an excellent and safe option for complex vascular reconstructions in patients with evidence of IVC invasion.

\section{Conflicts of Interest}

The Authors declare no conflicts of interest.

\section{Authors' Contributions}

$\mathrm{MCV}, \mathrm{AM}, \mathrm{SS}$ and GC designed the study. MCV, AM, SS, and GC wrote the article. MCV, AM, SS and GC collected the data. SS and GC offered scientific advice. MCV, AM, SS and GC revised the manuscript. GC critically revised the manuscript and was the supervisor.

\section{References}

1 Cohen HT and McGovern FJ: Renal-cell carcinoma. N Engl J Med 353(23): 2477-2490, 2005. PMID: 16339096. DOI: 10.1056/NEJMra043172

2 Zheng I, Alameddine M, Tan Y, Moghadamyeghaneh Z, Jue JS, Yusufali A, Farag A and Ciancio G: Collecting duct carcinoma of the native kidney in a renal transplant recipient. Case Rep Transplant 2017: 4527104, 2017. PMID: 29062581. DOI: $10.1155 / 2017 / 4527104$

3 Waqas M, Rahim W, Shohab D, Khawaja MA, Ali Z and Mamoon N: Primary renal epithelioid angiosarcoma. J Coll Physicians Surg Pak 28(3): S66-S68, 2018. PMID: 29482713. DOI: $10.29271 /$ jcpsp.2018.03.S66

4 Trpkov $\mathrm{K}$ and Hes $\mathrm{O}$ : New and emerging renal entities: a perspective post-WHO 2016 classification. Histopathology 74(1): 31-59, 2019. PMID: 30565301. DOI: 10.1111/his.13727

5 Hansel DE, Epstein JI, Berbescu E, Fine SW, Young RH and Cheville JC: Renal carcinoid tumor: a clinicopathologic study of 21 cases. Am J Surg Pathol 31(10): 1539-1544, 2007. PMID: 17895755. DOI: 10.1097/PAS.0b013e318042d596

6 Krishnan B, Truong LD, Saleh G, Sirbasku DM and Slawin KM: Horseshoe kidney is associated with an increased relative risk of primary renal carcinoid tumor. J Urol 157(6): 2059-2066, 1997. PMID: 9146580.

7 Bégin LR, Guy L, Jacobson SA and Aprikian AG: Renal carcinoid and horseshoe kidney: a frequent association of two rare entities - a case report and review of the literature. J Surg
Oncol 68(2): 113-119, 1998. PMID: 9624041. DOI: 10.1002/(sici)1096-9098(199806)68:2<113::aid-jso8>3.0.co;2-9

8 Ciancio G, Vaidya A, Savoie M and Soloway M: Management of renal cell carcinoma with level III thrombus in the inferior vena cava. J Urol 168(4 Pt 1): 1374-1377, 2002. PMID: 12352396. DOI: 10.1097/01.ju.0000023441.00587.02

9 Neves RJ and Zincke H: Surgical treatment of renal cancer with vena cava extension. Br J Urol 59(5): 390-395, 1987. PMID: 3594097. DOI: 10.1111/j.1464-410x.1987.tb04832.x

10 Ciancio G, Vaidya A and Soloway M: Early ligation of the renal artery using the posterior approach: a basic surgical concept reinforced during resection of large hypervascular renal cell carcinoma with or without inferior vena cava thrombus. BJU Int 92(4): 488-489, 2003. PMID: 12930447. DOI: 10.1046/j.1464410x.2003.04372.x

11 Ciancio G, Gonzalez J, Shirodkar SP, Angulo JC and Soloway MS: Liver transplantation techniques for the surgical management of renal cell carcinoma with tumor thrombus in the inferior vena cava: step-by-step description. Eur Urol 59(3): 401-406, 2011. PMID: 20724064. DOI: 10.1016/j.eururo.2010.07.028

12 Ciancio G, Hawke C and Soloway M: The use of liver transplant techniques to aid in the surgical management of urological tumors. J Urol 164(3 Pt 1): 665-672, 2000. PMID: 10953122. DOI: $10.1097 / 00005392-200009010-00012$

13 Warren AY and Harrison D: WHO/ISUP classification, grading and pathological staging of renal cell carcinoma: standards and controversies. World J Urol 36(12): 1913-1926, 2018. PMID: 30123932. DOI: 10.1007/s00345-018-2447-8

14 Jonasch E, Gao J and Rathmell WK: Renal cell carcinoma. BMJ 349: g4797, 2014. PMID: 25385470. DOI: 10.1136/bmj.g4797

15 Kennedy SM, Merino MJ, Linehan WM, Roberts JR, Robertson $\mathrm{CN}$ and Neumann RD: Collecting duct carcinoma of the kidney. Hum Pathol 21(4): 449-456, 1990. PMID: 2318486. DOI: 10.1016/0046-8177(90)90209-n

16 Srigley JR and Eble JN: Collecting duct carcinoma of kidney. Semin Diagn Pathol 15(1): 54-67, 1998. PMID: 9503506.

17 Kuroda N, Toi M, Hiroi M and Enzan H: Review of collecting duct carcinoma with focus on clinical and pathobiological aspects. Histol Histopathol 17(4): 1329-1334, 2002. PMID: 12371157. DOI: $10.14670 / \mathrm{HH}-17.1329$

18 Kobayashi N, Matsuzaki O, Shirai S, Aoki I, Yao M and Nagashima Y: Collecting duct carcinoma of the kidney: an immunohistochemical evaluation of the use of antibodies for differential diagnosis. Hum Pathol 39(9): 1350-1359, 2008. PMID: 18602672. DOI: 10.1016/j.humpath.2007.11.020

19 Pepek JM, Johnstone PA and Jani AB: Influence of demographic factors on outcome of collecting duct carcinoma: a Surveillance, Epidemiology, and End Results (SEER) database analysis. Clin Genitourin Cancer 7(2): E24-E27, 2009. PMID: 19692318. DOI: 10.3816/CGC.2009.n.017

20 Tokuda N, Naito S, Matsuzaki O, Nagashima Y, Ozono S, Igarashi T and Japanese Society of Renal Cancer: Collecting duct (Bellini duct) renal cell carcinoma: a nationwide survey in Japan. J Urol 176(1): 40-3; discussion 43, 2006. PMID: 16753362. DOI: 10.1016/S0022-5347(06)00502-7

21 Dason S, Allard C, Sheridan-Jonah A, Gill J, Jamshaid H, Aziz T, Kajal B and Kapoor A: Management of renal collecting duct carcinoma: a systematic review and the McMaster experience. Curr Oncol 20(3): e223-e232, 2013. PMID: 23737692. DOI: $10.3747 /$ co.20.1230 
22 Zhao RN, Nie LH, Gong R, Wang JZ, Wazir R, Liu LR, Song TR and Wei Q: Active targeted therapy for metastatic collecting duct carcinoma of the kidney: a case report and review of the literature. Int Urol Nephrol 45(4): 1017-1021, 2013. PMID: 23686671. DOI: 10.1007/s11255-013-0468-1

23 Omiyale AO and Carton J: Clinical and pathologic features of primary angiosarcoma of the kidney. Curr Urol Rep 19(2): 4 , 2018. PMID: 29383452. DOI: 10.1007/s11934-018-0755-6

24 Liu H, Huang X, Chen H, Wang X and Chen L: Epithelioid angiosarcoma of the kidney: A case report and literature review. Oncol Lett 8(3): 1155-1158, 2014. PMID: 25120677. DOI: $10.3892 /$ ol .2014 .2292

25 Hart $\mathrm{J}$ and Mandavilli S: Epithelioid angiosarcoma: a brief diagnostic review and differential diagnosis. Arch Pathol Lab Med 135(2): 268-272, 2011. PMID: 21284449. DOI: $10.5858 / 135.2 .268$

26 Iacovelli R, Orlando V, Palazzo A and Cortesi E: Clinical and pathological features of primary renal angiosarcoma. Can Urol Assoc J 8(3-4): E223-E226, 2014. PMID: 24839487. DOI: 10.5489/cuaj. 1585

27 Badiu CD, Rahnea Nita G, Ciuhu AN, Manea C, Smarandache CG, Georgescu DG, Bedereag SI, Cocosila CL, Braticevici B, Mehedintu $\mathrm{C}$ and Grigorean VT: Neuroendocrine renal carcinoma - Therapeutic and diagnostic issues. Acta Endocrinol (Buchar) 12(3): 355-361, 2016. PMID: 31149114. DOI: 10.4183/aeb.2016.355

28 Romero FR, Rais-Bahrami S, Permpongkosol S, Fine SW, Kohanim S and Jarrett TW: Primary carcinoid tumors of the kidney. J Urol 176(6 Pt 1): 2359-2366, 2006. PMID: 17085102. DOI: $10.1016 /$ j.juro.2006.07.129

29 Kojiro M, Ohishi $\mathrm{H}$ and Isobe $\mathrm{H}$ : Carcinoid tumor occurring in cystic teratoma of the kidney: a case report. Cancer 38(4): 16361640, 1976. PMID: 991082. DOI: 10.1002/1097-0142(1976 10)38:4<1636::aid-cncr2820380432>3.0.co;2-n
30 Shibata R, Okita H, Shimoda M, Asakura H, Murai M, Sakamoto M and Hata J: Primary carcinoid tumor in a polycystic kidney. Pathol Int 53(5): 317-322, 2003. PMID: 12713568. DOI: 10.1046/j.1440-1827.2003.01469.x

31 Shurtleff BT, Shvarts O and Rajfer J: Carcinoid tumor of the kidney: case report and review of the literature. Rev Urol 7(4): 229-233, 2005. PMID: 16985835.

32 Jain D, Sharma MC, Singh K and Gupta NP: Primary carcinoid tumor of the kidney: case report and brief review of literature. Indian J Pathol Microbiol 53(4): 772-774, 2010. PMID: 21045413. DOI: $10.4103 / 0377-4929.72087$

33 Hassan M, Ciancio G, Shirodkar SP, Del Mazo MB, Barron M, Salerno TA and Livingstone AS: Surgical technique of removal of inferior vena cava leiomyosarcoma extending into the right atrium without deep hypothermic circulatory arrest. J Card Surg 25(3): 277-281, 2010. PMID: 20149014. DOI: 10.1111/j.15408191.2009.00980.x

34 Delis SG, Madariaga $\mathrm{J}$ and Ciancio G: Combined liver and inferior vena cava resection for hepatic malignancy. J Surg Oncol 96(3): 258-264, 2007. PMID: 17443739. DOI: 10.1002/jso.20794

35 Quinones-Baldrich WJ and Farley S: Techniques for inferior vena cava resection and reconstruction for retroperitoneal tumor excision. J Vasc Surg Venous Lymphat Disord 1(1): 84-89, 2013. PMID: 26993901. DOI: 10.1016/j.jvsv.2012.10.056 